\title{
Theoretical aspects of several successive two-step redox mechanisms in protein-film cyclic staircase voltammetry
}

\author{
Rubin Gulaboski ${ }^{\mathrm{a}, *}$, Pavlinka Kokoškarova ${ }^{\mathrm{b}}$, Saša Mitrev ${ }^{\mathrm{a}}$ \\ a Department of Chemistry \& Department for plant and environment protection, Faculty of Agriculture, University “Goce Delčev”-Štip, Macedonia \\ b Department of Chemistry, Faculty of Natural Sciences and Mathematics, "Ss Kiril i Metodij" University, Skopje, Macedonia
}

\section{A R T I C L E I N F O}

\section{Article history:}

Received 31 October 2011

Received in revised form 17 February 2012

Accepted 22 February 2012

Available online 3 March 2012

\section{Keywords:}

Redox enzymes

Metalloproteins

Theoretical modeling

EE, ECE and EECat surface redox reactions

Protein-film voltammetry

\begin{abstract}
A B S T R A C T
Protein-film voltammetry (PFV) is a versatile tool designed to provide insight into the enzymes physiological functions by studying the redox properties of various oxido-reductases with suitable voltammetric technique. The determination of the thermodynamic and kinetic parameters relevant to protein's physiological properties is achieved via methodologies established from theoretical considerations of various mechanisms in PFV. So far, the majority of the mathematical models in PFV have been developed for redox proteins undergoing a single-step electron transfer reactions. However, there are many oxido-reductases containing quinone moieties or polyvalent ions of transition metals like Mo, Mn, W, Fe or Co as redox centers, whose redox chemistry can be described only via mathematical models considering successive two-step electron transformation. In this work we consider theoretically the protein-film redox mechanisms of the EE (Electrochemical-Electrochemical), ECE (Electrochemical-Chemical-Electrochemical), and EECat (Electrochemical-Electrochemical-Catalytic) systems under conditions of cyclic staircase voltammetry. We also propose methodologies to determine the kinetics of electron transfer steps by all considered mechanisms. The experimentalists working with PFV can get large benefits from the simulated voltammograms given in this work.
\end{abstract}

(c) 2012 Elsevier Ltd. All rights reserved.

\section{Introduction}

The voltammetric methods are used for more than 60 years to study the redox reactions of numerous small molecules and ions, and to provide access to relevant kinetic and thermodynamic information linked to various functions of considered compounds [1]. While the voltammetric experiments of small chemical systems are relatively easy to be performed, the transfer of electrons between the working electrode and large lipophilic molecules (such as lipophilic proteins, for example), however, is not an easy task, and it always suffers a number of drawbacks. The main cause for this is seen in the large inactive (insulating) part of the proteins that hinders significantly the direct electron exchange between the electrode and the redox active center of a given protein $[2,3]$. In the late 70 s of the last century the group led by Hill was first that performed direct voltammetry of several watersoluble cytochromes at solid electrodes [4]. The effectiveness of the direct protein voltammetry was further increased by making adsorption of the studied proteins as a monolayer on the bare or modified surfaces of suitable electrodes. The worldwide accepted name of this new methodology given by Armstrong is a protein

\footnotetext{
* Corresponding author.

E-mail address: rubin.gulaboski@ugd.edu.mk (R. Gulaboski).
}

film voltammetry (PFV). Since the establishment of PFV some 15 years ago [5], this technique became a versatile toll to study the redox transformation of scores of enzymes, while providing closer insight into the physiological functions of various oxidoreductases [6-10]. The basic principles of PFV consider initial adsorption of a given protein in a form of a monolayer on the surface of suitable electrode (preferably graphite). The protein adsorption at the electrode surface is achieved by its self-assembling from the protein-containing electrolyte solutions. Hitherto, protein film voltammetry benefits from its sensitivity due to the extremely small sample requirements. For example, monolayer coverage of a protein of $50,000 \mathrm{Da}$ requires just a few picomoles $/ \mathrm{cm}^{2}$ [5]. The PFV is a rather simple experimental methodology that provides access not only to the mechanism of redox transformation, but also to thermodynamic and kinetic parameters relevant to physiological features of many redox enzymes [2,3,5-10]. Moreover, this methodology is also extensively used as a versatile tool for simple detection of reactive oxygen species as hydrogen peroxide, hydroxyl and superoxide radicals $[2,3,5,6]$. The ability to investigate the redox protein reactivity with a given substrate over a wide range of conditions is enabled by studying the protein features with common voltammetric techniques such as cyclic or square-wave voltammetry [2-10]. In order to understand closely the mechanism of the enzyme-substrate interactions, and to have access to the thermodynamic and kinetic parameters 
relevant to those interactions, one must get a help of mathematical models developed for relevant enzymatic systems. So far, the majority of the theoretical models in PFV are developed for a single-step electron transfer systems under conditions of linear cyclic voltammetry-LCV [3,5-10]. Alongside, our group started developing theoretical models relevant to PFV under conditions of square-wave voltammetry (SWV) $[6,11-20]$. In the last few years we started modeling complex redox reactions of enzymes that can be interconverted from one oxidation step to other via two or more consecutive electron transfer steps [12,14,17]. Such enzymes are common in the biological systems, where one finds many classes of proteins containing electroactive centers that undergo redox transformations in several successive electron steps [21-24]. Of these, the most important are flavoproteins, then proteins with quinone moiety [21-23], and the proteins containing polyvalent ions of transition metals like Mo, Mn, W, Fe or Co as redox centers [24]. In this paper we present a theoretical study of three redox mechanisms that are relevant for redox proteins undergoing multiple successive electrochemical transformations under conditions of cyclic staircase voltammetry. The considered systems are assigned as EE (Electrochemical-Electrochemical), ECE (Electrochemical-Chemical-Electrochemical) and EECat (Electrochemical-Electrochemical-Catalytic) redox mechanisms. This study will help the experimentalists in elucidating the complex redox mechanisms of significant redox proteins, and it will give qualitative criteria for distinguishing particular mechanism from the similar ones. The current work is seen as a complementary to our previous works published elsewhere [12,14,17].

\section{Mathematical models}

The considered systems in this work are assigned as surface EE, ECE, and EECat redox mechanisms and these can be described by following reaction schemes:

\section{EE mechanism \\ Electrochemical step \#1: \\ Electrochemical step \#2: \\ II. ECE mechanism \\ Electrochemical step \#1: \\ Chemical step: \\ Electrochemical step \#2: III. EECat mechanism \\ Electrochemical step \#1: Electrochemical step \#2: \\ Catalytic step:}

$$
\begin{aligned}
& \mathrm{A}(\text { ads })+\text { ne }-\rightleftarrows \mathrm{B}(\text { ads }) \\
& \mathrm{B}(\text { ads })+\text { ne }-\rightleftarrows \mathrm{C}(\text { ads }) \\
& \\
& \mathrm{A}(\text { ads })+\text { ne }-\rightleftarrows \mathrm{B}(\text { ads }) \\
& \mathrm{B}(\text { ads })+\mathrm{Y} \stackrel{k_{\mathrm{f}}}{\longrightarrow} \mathrm{C}(\text { ads }) \\
& \mathrm{C}(\text { ads })+\text { ne }-\rightleftarrows \mathrm{D}(\text { ads }) \\
& \mathrm{A}(\text { ads })+\text { ne }-\rightleftarrows \mathrm{B}(\text { ads }) \\
& \mathrm{B}(\text { ads })+\text { ne }-\rightleftarrows \mathrm{C}(\text { ads }) \\
& \mathrm{C}(\text { ads })+\mathrm{Sub} \stackrel{k_{\text {cat }}}{\longrightarrow} \mathrm{B}(\text { ads })
\end{aligned}
$$

We assume that all redox active participants in the electrode mechanisms I-III are strongly adsorbed (ads) at the electrode surface, thus we neglected the mass transfer via diffusion in our modeling procedure. The symbol Y stays for an electrochemically inactive reactant, whose concentration is much higher than the initial concentration of all adsorbed electroactive species in the reaction mechanism II. Therefore, the chemical step in the reaction mechanism II is assumed to be of pseudo-first order. The physical parameter $k_{\mathrm{f}}\left(\mathrm{s}^{-1}\right)$ is the pseudo-first order rate constant of the chemical step in the mechanism II. This parameter is related to the bulk concentration of $\mathrm{Y}$ as follows $k_{\mathrm{f}}=k_{\mathrm{f}}^{\prime} c(\mathrm{Y})$, where $k_{\mathrm{f}}^{\prime}$ is the real chemical rate constant having units $\mathrm{mol}^{-1} \mathrm{~cm}^{3} \mathrm{~s}^{-1}$. By Sub we assign an electrochemically inactive substrate (or catalytic reagent) in reaction mechanism III. The bulk concentration of the substrate Sub present in electrochemical cell is supposed to be much higher than the initial concentration of all adsorbed electroactive species. Consequently, the chemical step in the reaction mechanism (III) is also considered to be of pseudo-first order. By $k_{\text {cat }}\left(s^{-1}\right)$ we define a pseudo first order catalytic rate constant that is related to the bulk concentration of the substrate via equation $k_{\text {cat }}=k_{\text {cat }}^{\prime} c(\operatorname{Sub})$.
In the last expression, $k_{\text {cat }}^{\prime}$ is the real chemical (i.e. catalytic or Michaelis-Menten) rate constant $\left(\mathrm{mol}^{-1} \mathrm{~cm}^{3} \mathrm{~s}^{-1}\right)$, while $c(\mathrm{Sub})$ is the molar concentration of the substrate Sub present in excess in the bulk solution in electrochemical cell. The electrode mechanisms I-III can be mathematically defined by the following set of equations:

A. For EE mechanism (redox mechanism I):

$$
\begin{aligned}
\frac{\mathrm{d} \Gamma(\mathrm{A})}{\mathrm{d} t} & =\frac{I_{1}}{n F S} \\
\frac{\mathrm{d} \Gamma(\mathrm{B})}{\mathrm{d} t} & =-\frac{I_{1}}{n F S}+\frac{I_{2}}{n F S} \\
\frac{\mathrm{d} \Gamma(\mathrm{C})}{\mathrm{d} t} & =-\frac{I_{2}}{n F S}
\end{aligned}
$$

B. For ECE mechanism (redox mechanism II):

$$
\begin{aligned}
& \frac{\mathrm{d} \Gamma(\mathrm{A})}{\mathrm{d} t}=\frac{I_{1}}{n F S} \\
& \frac{\mathrm{d} \Gamma(\mathrm{B})}{\mathrm{d} t}=-\frac{I_{1}}{n F S}-k_{\mathrm{f}} \Gamma(\mathrm{B}) \\
& \frac{\mathrm{d} \Gamma(\mathrm{C})}{\mathrm{d} t}=\frac{I_{2}}{n F S}+k_{\mathrm{f}} \Gamma(\mathrm{B}) \\
& \frac{\mathrm{d} \Gamma(\mathrm{D})}{\mathrm{d} t}=-\frac{I_{2}}{n F S}
\end{aligned}
$$

C. For EECat mechanism (redox mechanism III)

$$
\begin{aligned}
& \frac{\mathrm{d} \Gamma(\mathrm{A})}{\mathrm{d} t}=\frac{I_{1}}{n F S} \\
& \frac{\mathrm{d} \Gamma(\mathrm{B})}{\mathrm{d} t}=-\frac{I_{1}}{n F S}+\frac{I_{2}}{n F S}+k_{\mathrm{cat}} \Gamma(\mathrm{C}) \\
& \frac{\mathrm{d} \Gamma(\mathrm{C})}{\mathrm{d} t}=-\frac{I_{2}}{n F S}-k_{\mathrm{cat}} \Gamma(\mathrm{C})
\end{aligned}
$$

Eqs. (1)-(10) have been solved under the following initial and boundary conditions:

$t=0 ; \quad \Gamma(\mathrm{A})=\Gamma(\mathrm{A})^{*} ; \quad \Gamma(\mathrm{B})=\Gamma(\mathrm{C})=\Gamma(\mathrm{D})=0$

$t>0 ; \quad \Gamma(\mathrm{A})+\Gamma(\mathrm{B})+\Gamma(\mathrm{C})+\Gamma(\mathrm{D})=\Gamma(\mathrm{A})^{*}$

We assume that at the beginning of the experiment there is only compound A present in the cell, and it is strongly adsorbed at the electrode surface. The compound $C$ is generated chemically via chemical transformation of B with Y (for ECE mechanism), or electrochemically via redox transformation of B (for EE and EECat mechanisms). The compound $B$ is generated via electrochemical transformation of compound A (for all considered mechanisms), but also via catalytic regeneration reaction of the electrochemically generated product $C$ with the substrate Sub (for EECat mechanism). The term "catalytic" describes that the chemical (catalytic) reaction regenerates $B$ from $C$ by the EECat mechanism (III). The symbols $\Gamma(\mathrm{A}), \Gamma(\mathrm{B}), \Gamma(\mathrm{C})$ and $\Gamma(\mathrm{D})$ represent the surface concentrations of the species $\mathrm{A}, \mathrm{B}, \mathrm{C}$ and $\mathrm{D}$, respectively, while $\Gamma(\mathrm{A})^{*}$ is the total surface concentration of all species. $\Gamma$ is a symbol of the surface concentration of particular specie in the considered mechanisms that is function of the time $t$. $I$ is the symbol of the current, $S$ is the electrode surface area, $F$ is the Faraday constant, while $n$ is a number of exchanged electrons in an act of electrochemical transformation (it is assumed that $n$ are equal for both electrochemical steps in reaction mechanism I-III). The solutions of Eqs. (1)-(10) were obtained by help of Laplace transformations. The solutions for the surface concentrations of the electroactive species A, B, C and D in their integral forms for EE, ECE, and EECat read: 
A. For EE reaction mechanism (I)

$$
\begin{aligned}
& \Gamma(\mathrm{A})=\Gamma(\mathrm{A})^{*}-\int_{0}^{t} \frac{I_{1}(\tau)}{n F S} \mathrm{~d} \tau \\
& \Gamma(\mathrm{B})=-\int_{0}^{t} \frac{I_{1}(\tau)}{n F S} \mathrm{~d} \tau+\int_{0}^{t} \frac{I_{2}(\tau)}{n F S} \mathrm{~d} \tau \\
& \Gamma(\mathrm{C})=-\int_{0}^{t} \frac{I_{2}(\tau)}{n F S} \mathrm{~d} \tau
\end{aligned}
$$

B. For ECE reaction mechanism (II)

$$
\begin{aligned}
\Gamma(\mathrm{A})= & \Gamma(\mathrm{A})^{*}-\int_{0}^{t} \frac{I_{1}(\tau)}{n F S} \mathrm{~d} \tau \\
\Gamma(\mathrm{B})= & -\int_{0}^{t} \frac{I_{1}(\tau)}{n F S} \exp \left[-k_{\mathrm{f}}(t-\tau)\right] \mathrm{d} \tau \\
\Gamma(\mathrm{C})= & -\int_{0}^{t} \frac{I_{1}(\tau)}{n F S} \mathrm{~d} \tau+\int_{0}^{t} \frac{I_{1}(\tau)}{n F S} \exp \left[-k_{\mathrm{f}}(t-\tau)\right] \mathrm{d} \tau \\
& +\int_{0}^{t} \frac{I_{2}(\tau)}{n F S} \mathrm{~d} \tau \\
\Gamma(\mathrm{D})= & -\int_{0}^{t} \frac{I_{2}(\tau)}{n F S} \mathrm{~d} \tau
\end{aligned}
$$

C. For EECat reaction mechanism (III)

$$
\begin{aligned}
& \Gamma(\mathrm{A})=\Gamma(\mathrm{A})^{*}-\int_{0}^{t} \frac{I_{1}(\tau)}{n F S} \mathrm{~d} \tau \\
& \Gamma(\mathrm{B})=-\int_{0}^{t} \frac{I_{1}(\tau)}{n F S} \mathrm{~d} \tau+\int_{0}^{t} \frac{I_{2}(\tau)}{n F S} \exp \left[-k_{\text {cat }}(t-\tau)\right] \mathrm{d} \tau \\
& \Gamma(\mathrm{C})=-\int_{0}^{t} \frac{I_{2}(\tau)}{n F S} \exp \left[-k_{\text {cat }}(t-\tau)\right] \mathrm{d} \tau
\end{aligned}
$$

Further, we assume that the Buttler-Volmer formalism [1] applies at the electrode surface. In such case, the following conditions are valid at the electrode surface:

$$
\frac{I_{1}}{n F S}=k_{\mathrm{s}, 1} \exp \left(-\alpha \phi_{1}\right)\left[\Gamma(\mathrm{A})-\exp \left(\phi_{1}\right) \Gamma(\mathrm{B})\right]
$$

(holds for all mechanisms I-III)

$$
\frac{I_{2}}{n F S}=k_{\mathrm{s}, 2} \exp \left(-\alpha \phi_{2}\right)\left[\Gamma(\mathrm{B})-\exp \left(\phi_{2}\right) \Gamma(\mathrm{C})\right]
$$

(holds for mechanisms I and III)

$$
\frac{I_{2}}{n F S}=k_{\mathrm{s}, 2} \exp \left(-\alpha \phi_{2}\right)\left[\Gamma(\mathrm{C})-\exp \left(\phi_{2}\right) \Gamma(\mathrm{D})\right]
$$

(holds only for mechanism II)

Therefore, by substituting the corresponding Eqs. (13)-(22) into the relevant Buttler-Volmer expressions (23)-(25), we obtain integral forms of the mathematical solutions for EE, ECE and EECat surface mechanisms under chronoamperometric conditions. The numerical solutions of all mechanisms were obtained by using the method of Nicholson described elsewhere [25]. The numerical solutions of all considered mechanisms under conditions of squarewave voltammetry are given in our previous works published elsewhere $[6,12,14,17]$. It should be pointed out that the numerical solutions are formally equivalent for both square-wave and cyclic staircase voltammetry. However, the dimensionless parameters existing in the numerical solutions are defined differently for each technique. In Eqs. (23)-(25) $k_{\mathrm{s}, 1}\left(\mathrm{~s}^{-1}\right)$ and $k_{\mathrm{s}, 2}\left(\mathrm{~s}^{-1}\right)$ are the heterogenous rate constant of electron transfer corresponding to the standard redox potential of first $E_{\mathrm{A} / \mathrm{B}}^{\Theta}$ and second $E_{\mathrm{B} / \mathrm{C}}^{\Theta}$ electron transfer steps of the electrode mechanisms (I-III), $\alpha$ is the cathodic electron transfer coefficient, while $\phi_{1}=(n F / R T)\left(E-E_{\mathrm{A} / \mathrm{B}}^{\ominus}\right)$ and $\phi_{2}=$ $(n F / R T)\left(E-E_{\mathrm{B} / \mathrm{C}}^{\Theta}\right)\left[\right.$ or $\phi_{2}=(n F / R T)\left(E-E_{\mathrm{C} / \mathrm{D}}^{\Theta}\right)$ for EECat] are the relative dimensionless electrode potentials. All the potentials of the simulated voltammograms are referred vs. the standard redox potential of the first electrochemical step $E_{\mathrm{A} / \mathrm{B}}^{\ominus}$. For the technique considered in this work (cyclic staircase voltammetry), the following definitions are valid: by $\Psi_{1}=I_{1} \tau / n F S \Gamma^{*}$ and $\Psi_{2}=I_{2} \tau / n F S \Gamma^{*}$ we define the dimensionless currents for the first and the second redox step in mechanisms (I-III), $K \mathrm{I}=k_{\mathrm{s}, 1} \tau$ and $K \mathrm{II}=k_{\mathrm{s}, 2} \tau$ are the dimensionless redox kinetic parameters relevant to the first and the second electron transfer steps in reaction mechanisms (I-III), $\lambda=k_{\mathrm{f}} \tau$ is the chemical kinetic parameter relevant to the chemical step in the reaction mechanism II (for ECE reaction), while $\gamma=k_{\mathrm{c}} \tau$ is the dimensionless catalytic parameter corresponding to the chemical step in reaction mechanism III (for EECat reaction). With $\tau$ we define the duration of the single potential step of the potential ramp in the cyclic staircase signal. The excitation signal used in SCV consists of a staircase potential ramp, which is characterized by the duration of the potential tread $\tau$ and the step of the staircase ramp $\mathrm{d} E$. For numerical simulation, we used a time increment of $d=\tau / 25$. For the sake of simplicity, we assumed that the number of exchanged electrons $(n)$ and the electron transfer coefficients $(\alpha)$ of both redox steps in all considered mechanisms are equal. These parameters were set to $n=1$, and $\alpha=0.5$ in all simulations of the studied twostep redox mechanisms. All cyclic voltammograms are recorded by setting the initial potential to more positive values, and making a scan toward the switching potential having more negative values. The theoretical calculations have been performed by using the MATHCAD software. A detailed description of the simulation procedure of relevant surface mechanisms in MATHCAD program under conditions of SWV is provided in our previous works [14], and one can use those files for free.

\section{Results}

Cyclic voltammetry (CV) is a potentiodynamic electrochemical technique that is a versatile experimental tool in various laboratories $[1,26]$. In the modern potentiostats there are mainly two types of cyclo-voltammetric techniques: the classical linear cyclic voltammetry that is present in older potentiostas models, and the cyclic staircase voltammetry that is nowadays present in all modern potentiostats. The differences between these techniques exist in the form of the potential ramp, but also in the measuring manner [1]. In the linear cyclic voltammetry the working electrode potential is ramped linearly vs. time [1,26-28]. When the potential ramp in cyclic voltammetry reaches a given end value, then the potential ramp gets inverted. The cyclic staircase voltammetry method is a particular format of linear cyclic voltammetry, where a ramp with multiple potential steps is applied to the working electrode, and the response of the cell is measured at the end of each step. This measuring manner allows significant diminishment of the capacitive current (arising from the double layer charging) [1,29-32]. A comparison of the potential forms in linear and staircase cyclic voltammetry is given in Fig. $1 \mathrm{a}$ and $\mathrm{b}$, respectively. In our previous works we have treated the considered redox mechanisms under conditions of square-wave voltammetry [12,14,17]. In those works we have shown that the features of calculated voltammograms of surface EE, ECE, and EECat redox mechanisms strongly depend on one virtual thermodynamic parameter (or standard potential difference) " $\Delta E^{\ominus}$ " that is defined as a difference between the standard redox potentials of the second and the first redox 

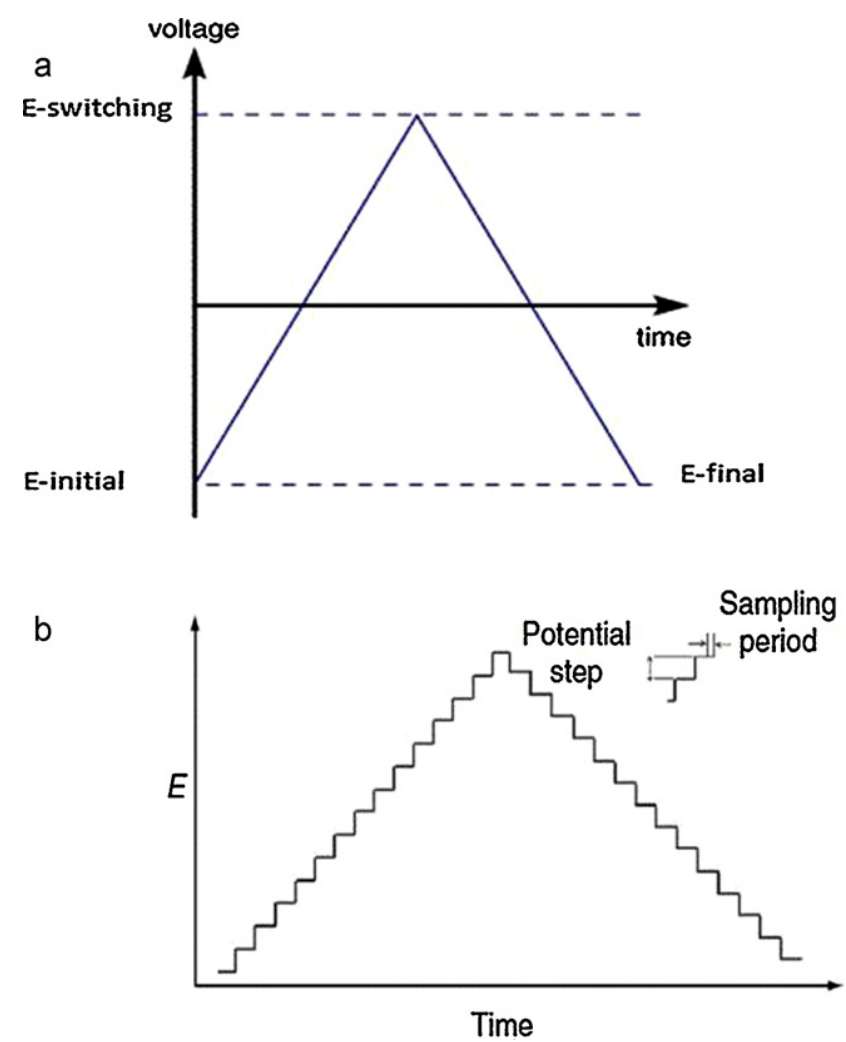

Fig. 1. Form of the potential ramps in linear cyclic voltammetry (a) and cyclic staircase voltammetry (b).

process, i.e. $\Delta E^{\Theta}=E_{\mathrm{B} / \mathrm{C}}^{\Theta}-E_{\mathrm{A} / \mathrm{B}}^{\Theta}$ (for EE and EECat mechanisms) or $\Delta E^{\Theta}=E_{\mathrm{C} / \mathrm{D}}^{\Theta}-E_{\mathrm{A} / \mathrm{B}}^{\Theta}$ (for ECE mechanism). Therefore, we consider two different situations in respect to the value of $\Delta E^{\ominus}$ : (a) the case where $\Delta E^{\ominus} \geq 0$, i.e. the situation in which the standard redox potential of the second redox process is equal or more positive than that of the first electron transfer step; and (b) case were $\Delta E^{\Theta} \leq-100 \mathrm{mV}$, which corresponds to scenario where the energy of the second electron transfer step is for at least $-100 \mathrm{mV}$ or more higher than that of the first electron transfer step.

\subsection{Energy of the second reduction step is lower or equal than that of the first reduction step, i.e. $\Delta E^{\ominus} \geq 0$}

In the biological systems, the EE mechanism occurs very rarely. In fact, the redox reaction of many metalloporteins and proteins with quinone moieties involve successive electron steps that are (almost always) inevitably coupled with protonation reactions. Thus, such experimental systems should be described more precisely by ECE, or ECEC models. However, in case that the protonation step is very fast in respect to the measuring time in voltammetry, then the entire mechanism can be approximated by EE redox mechanism. In situation of $\Delta E^{\Theta} \geq 0$ by the surface EE mechanism a quite interesting situation is observed if the kinetics of the first electron transfer step is one order of magnitude or more smaller than the kinetics of the second redox step (Fig. 2). Under such circumstances, two reduction peaks are observed in the forward scan, and only one broad peak in the reversal scan. The first pair of peaks at more positive potential is associated to the first redox step, and it possesses quasireversible voltammetric features, having peak to peak separation of more than $150 \mathrm{mV}$ (Fig. 2a). The peak-to-peak separation permanently diminishes as the value of the kinetic parameter for the first redox step increases. On the other hand, although the kinetics of the second reduction step is set to be bigger than that of the first electron transfer step, the peak associated to this redox process occurs at more negative potentials if $K \mathrm{I}<0.5$ (Fig. $2 \mathrm{a}-\mathrm{c}$ ). This is because the material for the second redox process comes only via the electrochemical transformation of the initial material $A$ in the first electron transfer step. Consequently, the lower kinetics of the first electron transfer step will produce less electroactive material that will be available for the second redox transformation, so more energy is needed to cause a second electron transfer. If, however, the dimensionless kinetic parameter of the first electron transfer step is $K I>0.5$, then one observes a single cyclic voltammogram in which both electron transfer processes of the first and the second redox steps have merged (Fig. $2 \mathrm{~d}$ ). Indeed, much more situations by this redox mechanism can be simulated by taking different values of $K \mathrm{I}$ and $K \mathrm{II}$ dimensionless parameters. This, however, will be very confusing for the readers, so we skip from further elaboration of more complicated situations by the EE mechanism. We advise the readers to our work [12] in order to get full insight into the features of this mechanism studied under conditions of SWV.

The more precise mechanism to describe the features of lipophilic redox proteins undergoing successive electron transformations is the ECE mechanism. By the surface ECE redox mechanism, a relevant parameter that deserves to be discussed is the value of the dimensionless chemical parameter $\lambda$. This parameter reflects the effect of the kinetics of the follow up chemical reaction to the features of simulated cyclic voltammograms. Shown in Fig. 3a-d are cyclic staircase voltammograms of an ECE mechanism for situation $\Delta E^{\Theta} \geq 0$, simulated for four different values of the dimensionless chemical parameter $\lambda$. As expected, for low values of the chemical parameter $(\lambda<0.005)$ the calculated cyclic voltammograms feature a single pair of peaks (Fig. 3a). In such situation, there are conditions only for the first electron transfer step to happen with significant rate. Because the rate of the chemical reaction is very slow, it cannot supply electroactive material for the second reduction step to occur during the measuring time of the voltammetric experiment. Therefore, the second electron transfer cannot happen with significant rate. However, as the value of the chemical parameter $\lambda$ gets values higher than 0.05 , then the peak in the reversal scan starts to become broader (Fig. 3b), and it results into two separated peaks as the chemical parameter $\lambda>0.1$ (Fig. $3 \mathrm{c}$ and d). Obviously, the intensity of the oxidation peak in reversal scans appearing at more positive potentials diminishes significantly as the value of chemical parameter $\lambda$ increases. This phenomenon is linked to the higher rate of conversion of the product of the first electron transfer step B into the starting material (C) that acts as a reactant in the second redox process [14]. Apparently, the features of EE and ECE mechanisms are quite different in situation of $\Delta E^{\Theta} \geq 0$ (compare Figs. 2 and 3, for example). These differences can be explored as qualitative criteria to distinguish between EE and ECE mechanisms.

A quite different behavior of the features of calculated voltammograms compared to those of EE and ECE mechanisms is seen by the surface EECat mechanism. A set of calculated cyclic voltammograms of the EECat mechanism is given in Fig. 4a-d. The calculated cyclic voltammograms in Fig. 4a-d portray the effect of the dimensionless catalytic parameter $\gamma$, when both dimensionless kinetic parameters of electron transfers $K \mathrm{I}$ and $K \mathrm{II}$ fall within the region of quasireversible electron transfer. One remarkable feature typical for the EECat mechanism is the notable rising of both branches of the cathodic and anodic current components at more negative potentials, i.e. in the potential regions after the Faradaic process occurs. This phenomenon appears as a consequence of continuous effect of the catalytic reaction that supplies permanently electroactive material $\mathrm{B}$ during the measuring time of the experiment $[1,6,11,17]$. This contributes to higher currents in the post-Faradaic potential region. Another notable feature by the surface EECat mechanism is seen in the reversal branch of the current: it gets the 

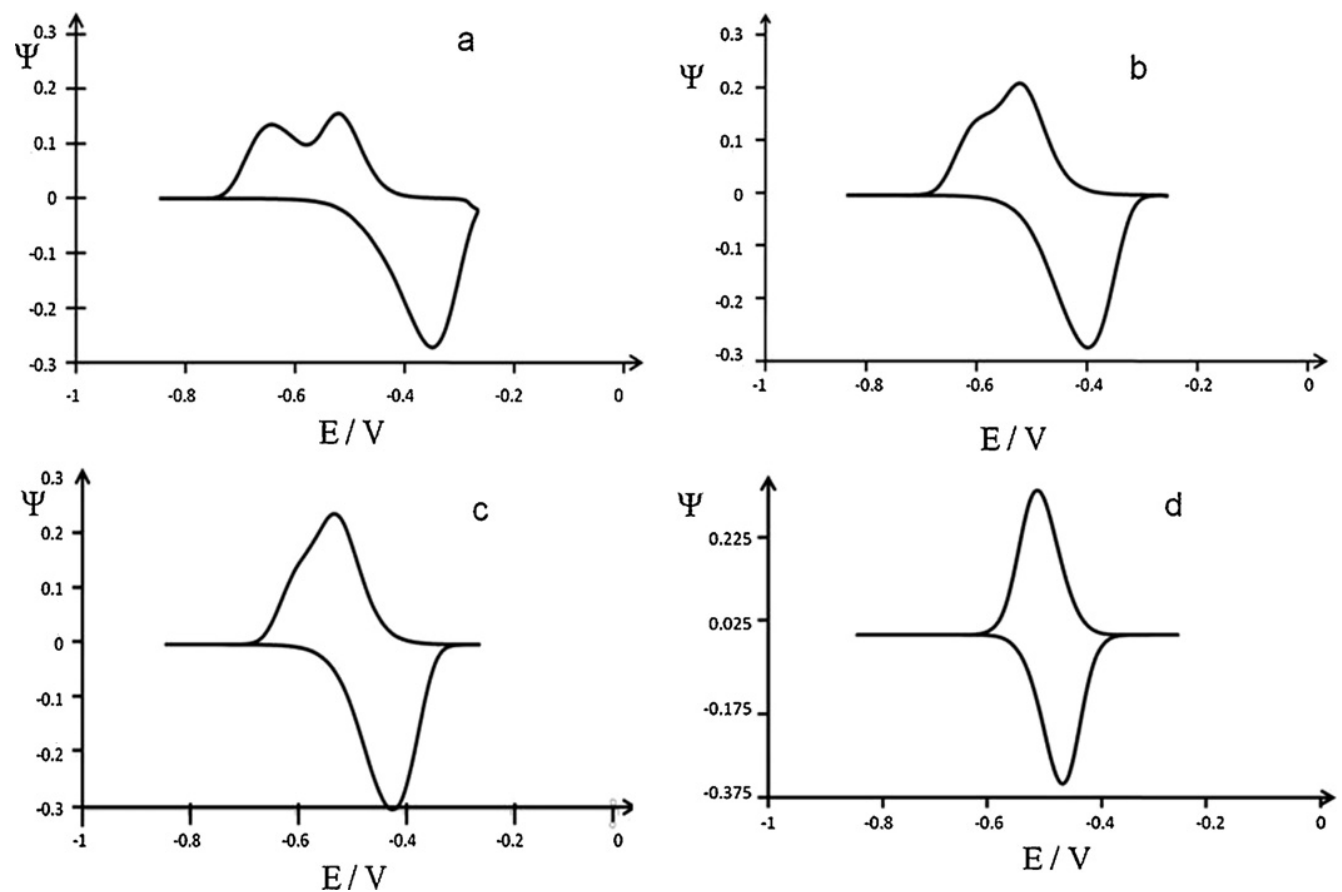

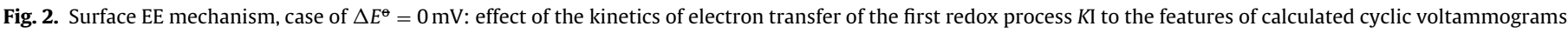

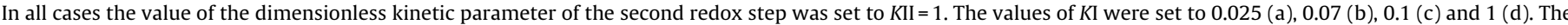

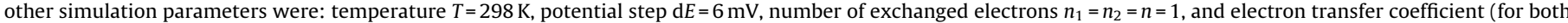
redox steps) $\alpha=0.5$.

same sign as the forward current in the potential regions after the Faradaic voltammetric response appears (see Fig. 4d). This effect in cyclic staircase voltammetry appears when the catalytic parameter $\gamma$ gets values higher than $\sim 0.1$. Under these conditions, the rate of the catalytic reaction is quite big, hence causing immediate transformation of the entire amount of reduced species $C$ back to the oxidized form B. Consequently, during the time scale of the voltammetric experiment the reoxidation process gets significantly hindered, and one observes a sigmoidal shape of the resulting voltammograms (Fig. 4d). Obviously, these features of the theoretical voltammograms of the EEcat redox mechanism are quite different than those of the EE and ECE mechanisms.
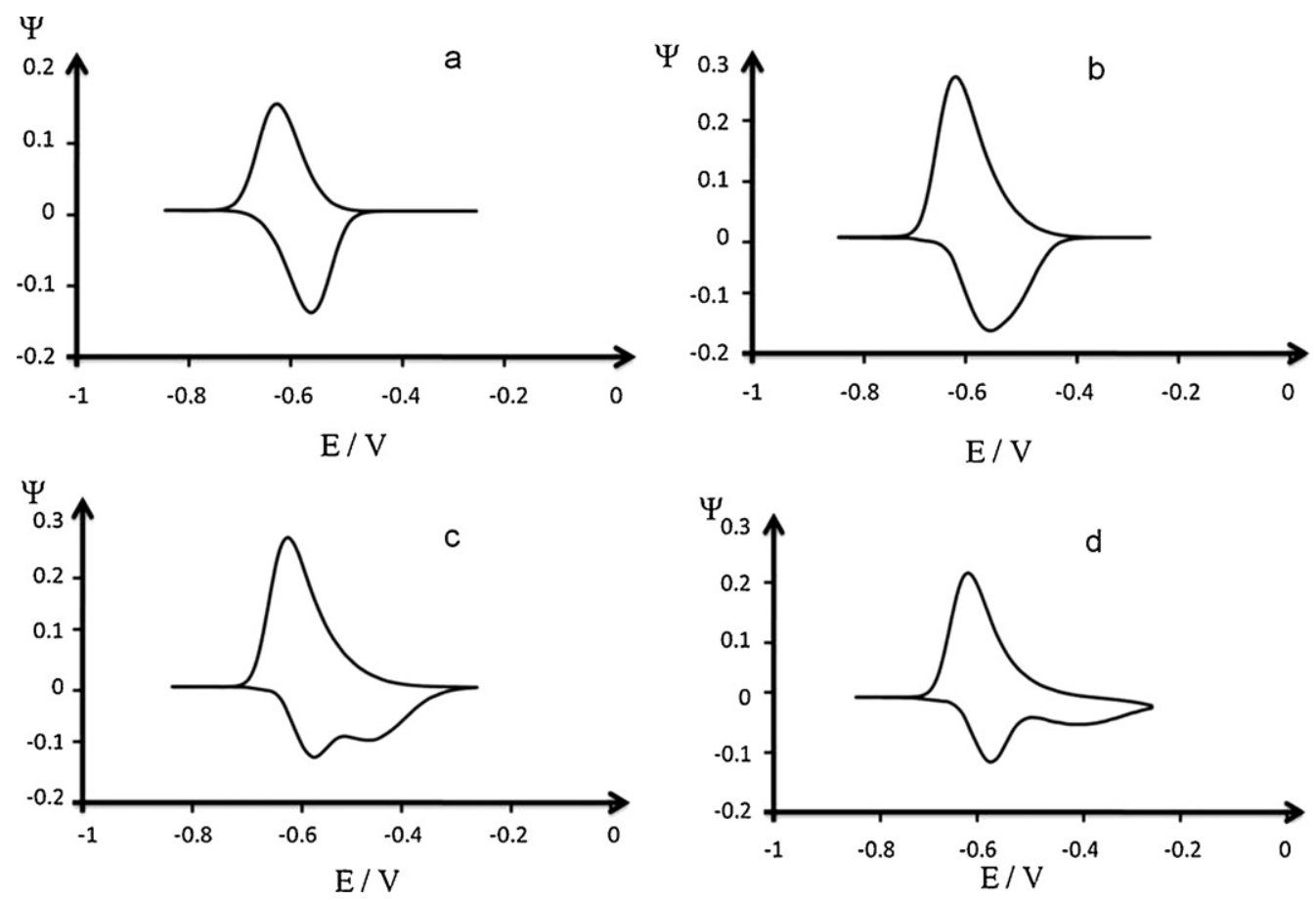

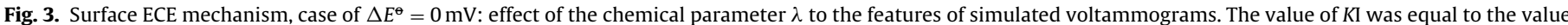

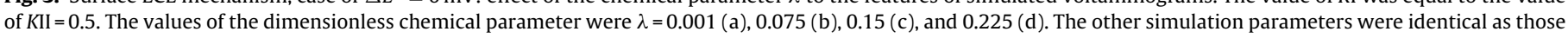
in Fig. 2. 


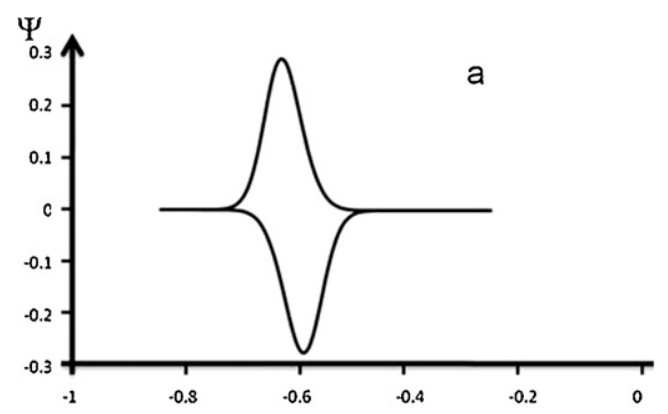

$\mathrm{E} / \mathrm{V}$

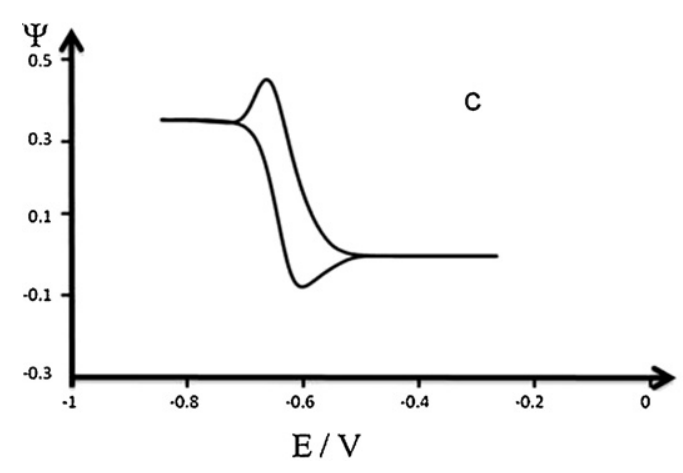

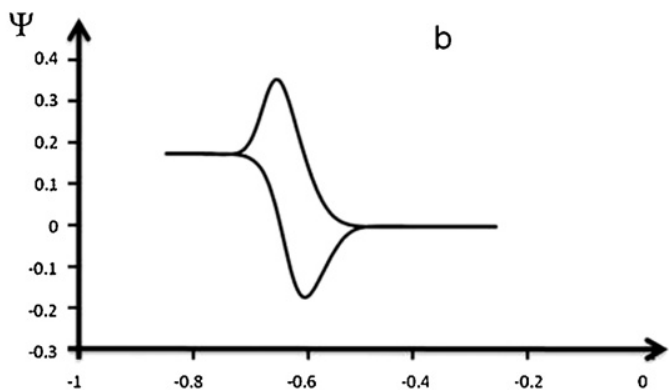

$\mathrm{E} / \mathrm{V}$

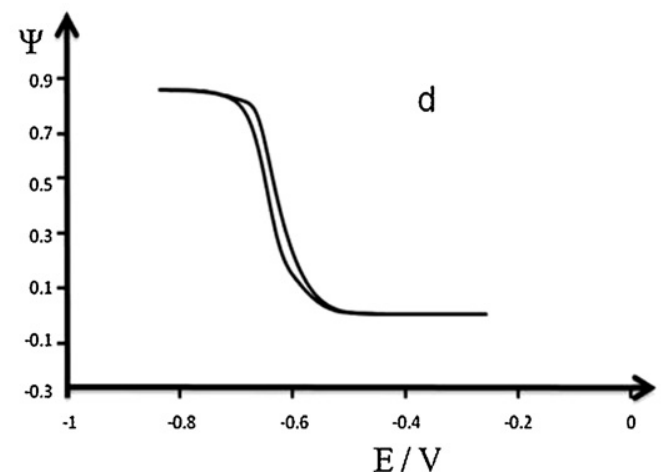

Fig. 4. Surface EECat mechanism, case of $\Delta E^{\ominus}=0 \mathrm{mV}$ : effect of the catalytic parameter $\gamma$ to the features of simulated voltammograms. The value of $K I$ was equal to the value of $K I I=1$. The values of the dimensionless catalytic parameter were $\gamma=0.001$ (a), 0.1 (b), $0.2(\mathrm{c})$, and 0.5 (d). The other simulation parameters were identical as those in Fig. 2 .

\subsection{Energy of the second reduction step is higher for $-100 \mathrm{mV}$} than that of the first reduction step, i.e. $\Delta E^{\ominus} \leq-100 \mathrm{mV}$

If the energy for occurrence of the second electron transfer step is for $-100 \mathrm{mV}$ or more higher than that of the first electron transfer step, then we observe well-resolved pair of peaks. Shown in
Fig. 5a-d are cyclic voltammograms of the EE mechanism simulated for $K \mathrm{I}=1$ and $\Delta E^{\Theta}=-100 \mathrm{mV}$, and four different values of the kinetic parameter of the second redox step KII. Under such conditions, the cyclo-voltammetric responses feature two cathodic and two anodic signals. As the dimensionless kinetic parameter KII gets values in the quasireversible region i.e. from 0.1 to 1.5 , an increase
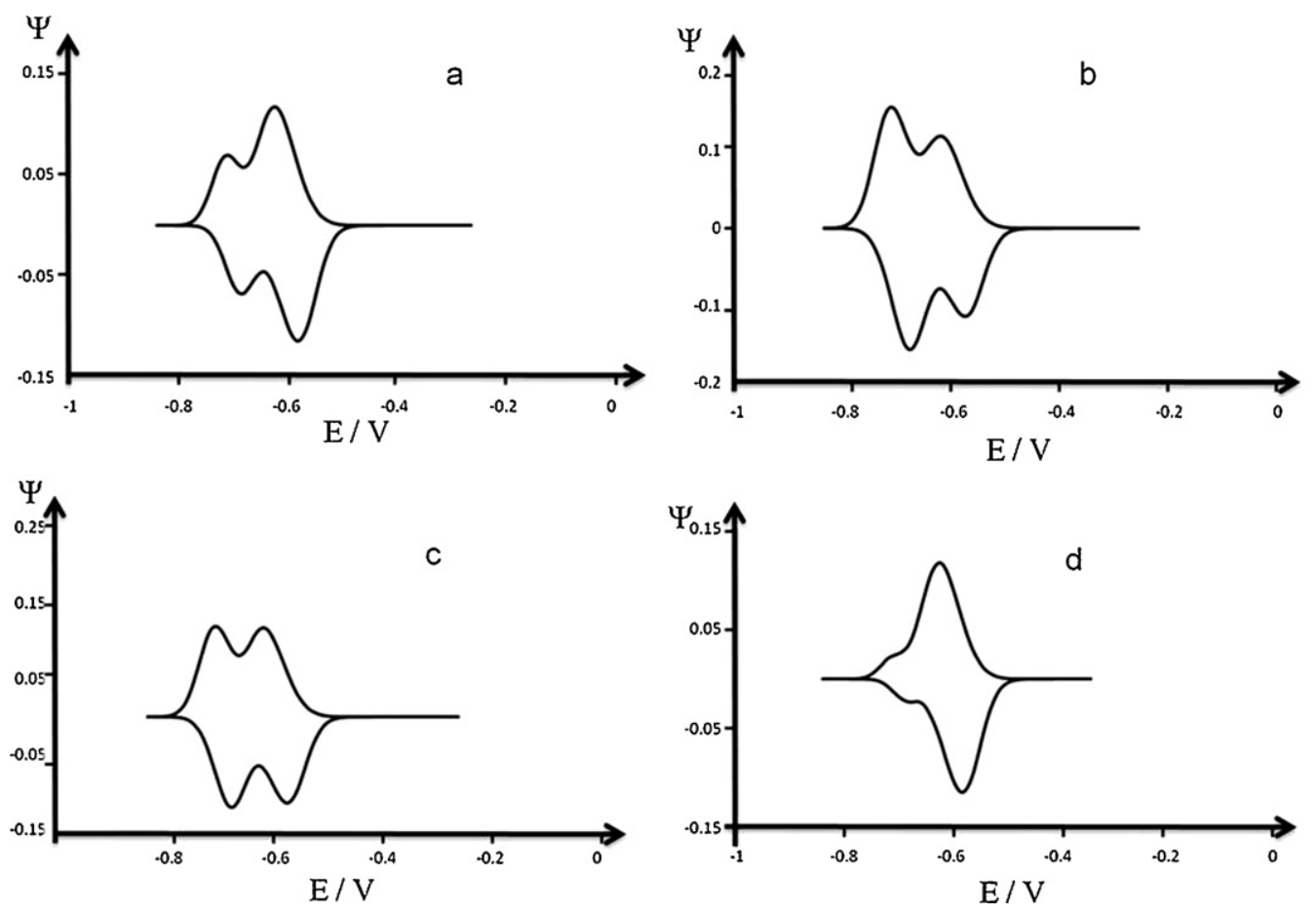

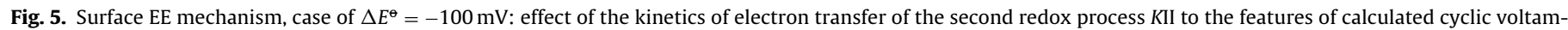

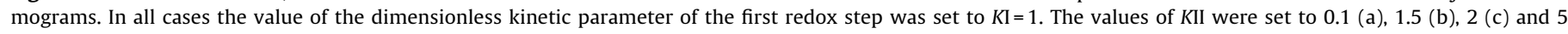
(d). 
of the peak currents of both cathodic and anodic current components of the second redox process at more negative potentials is observed (Fig. 5a and b). However, if the value of KII>1.5 (in the reversible region of electron transfer) then both current components of the second redox process start diminishing by increasing of $K \mathrm{II}$ (Fig. $5 \mathrm{c}$ and d). This phenomenon is due to the measuring manner of the pulse voltammetric techniques by the surface confined redox reaction, and it is explained in more details elsewhere [31]. Shortly, the pulse voltammetric techniques measure the current in the small time-interval at the end of the imposed pulses. The highest currents will be detected by quasireversible surface systems due to the synchronization of the rate of electron transfer with the measuring time interval in which the current is measured. This contributes to the multiple use of the redox material during measuring time, thus resulting in bigger measured currents. If the kinetic of electron transfer is, however, very high, then the majority of $B$ will be converted to $C$ at the beginning of the imposed potential pulses (in this interval the current is not being measured). Consequently, less material will be available to be detected at the end of the pulses where the current is measured, and smaller currents will be measured by fast or reversible redox process. More details about these phenomena can be found elsewhere [6,31].

The effect of the chemical parameter $\lambda$ to the features of calculated cyclic voltammograms of the surface ECE mechanism for $\Delta E^{\ominus}=-150 \mathrm{mV}$ is shown in Fig. 6a-d. If the kinetic of the first redox step falls within the quasireversible region of electron transfer, then a single voltammetric response is observed if the value of the chemical kinetic parameter $\lambda<0.001$ (Fig. 6a). This is because the chemical step is very slow and there will be slow conversion of B to C. Consequently, there will be no significant amount of material $\mathrm{C}$ created to undergo a second electron transfer during the experimental measuring time. As the chemical parameter gets values $\lambda>0.01$, a second redox process located at more negative potentials is observed that gains in its intensity by increasing the value of $\lambda$ (Fig. 6b-d). This phenomenon observed by the second redox transfer step is associated with comparable increase of the chemical irreversibility of the first redox process. Since the product of the first redox process (B) is "consumed" i.e. converted to $C$ via chemical transformation, the peak current of the backward (anodic) component of the cyclic voltammogram diminishes proportionally with the increasing of the value of $\lambda$ (see the anodic current component of the voltammetric process at more positive potentials in Fig. 6b-d). The decrease of the anodic peak current of the first redox step is also associated with the increase of the peak-to-peak separation by the response of the first redox process. This is a typical feature for the occurrence of the chemical reaction to the product of the first electron transfer step [6,14,27-30]. The features of the cyclic voltammograms shown in Fig. 6a-d can serve as simple qualitative criteria for recognizing the ECE mechanism, and for distinguishing it from the EE mechanism.

The third considered mechanism in this work (the surface EECat mechanism) again possesses voltammetric features that easily distinguish it from the EE and ECE redox mechanisms. The cyclic voltammograms presented in Fig. $7 a-d$ show the effect of the dimensionless catalytic parameter $\gamma$ to the features of the calculated curves for situation of $\Delta E^{\ominus}=-125 \mathrm{mV}$, when both $K \mathrm{I}$ and $K$ II fall within the quasireversible region of electron transfer. The current voltage profile at panel (a) of Fig. 7 corresponds to the case where no substrate ( $\mathrm{Sub}$ ) has been added to the solution, and therefore no chemical reaction occurs. However, as the catalytic agent (Sub) is added in the solution, then the catalytic reaction begins. For a given scan rate and concentration of Sub, the current of the second redox step will start to get higher values than that measured in absence of catalytic agent. This effect is due to the fact that the reactant of the second electron transfer step $B$ is being regenerated during the measuring time of the cyclo-voltammetric experiment, and can therefore react again at the electrode surface. As the concentration of the catalytic agent Sub is increased, the cathodic current of the second redox step also increases since more conversion of $\mathrm{C}$ to $\mathrm{B}$ occurs via the chemical reaction. This increase of the cathodic current of the second redox step is normally associated with a concomitant decrease of its anodic current components
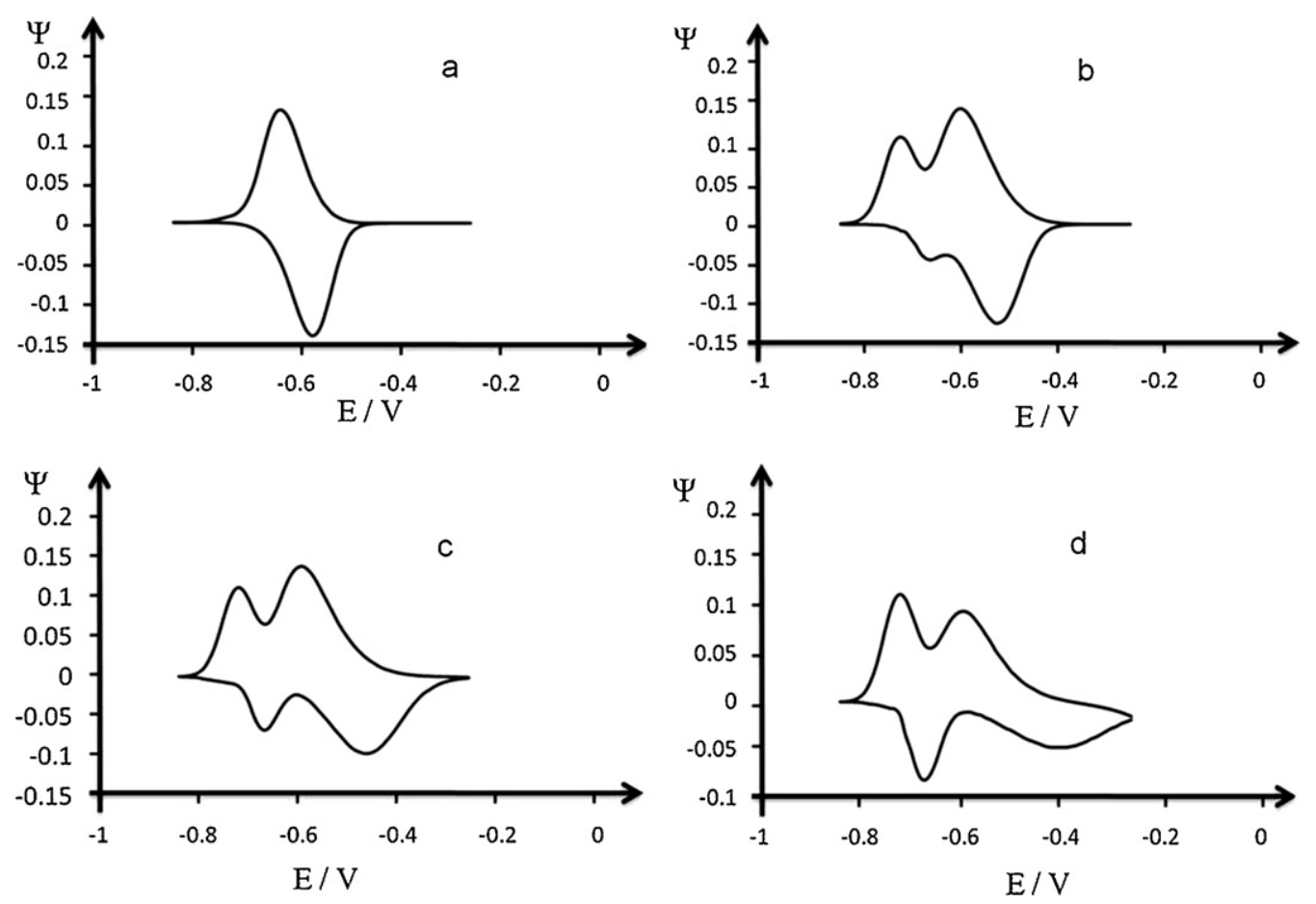

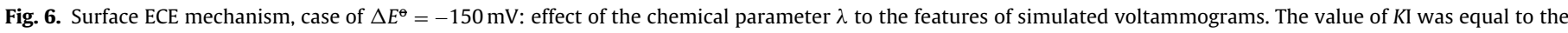

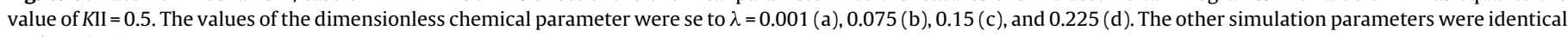
as those in Fig. 2. 

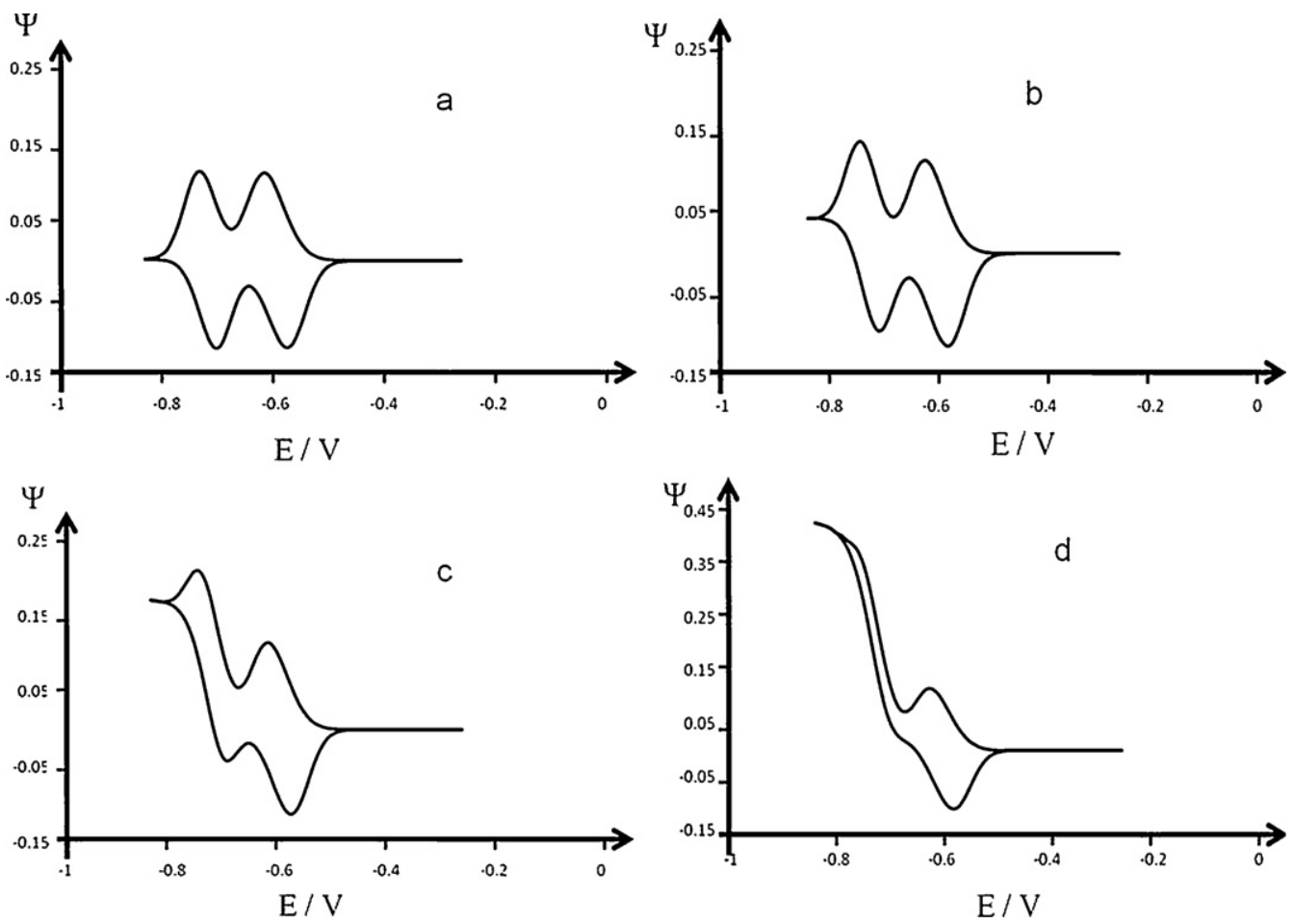

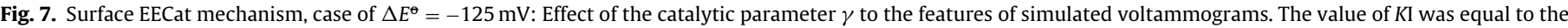

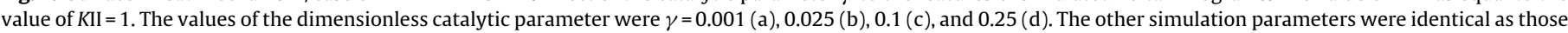
in Fig. 2.

(see Fig. $7 b$ and c). As the value of catalytic parameter reaches a value higher than $\gamma>0.2$, then a sigmoidal-type of voltammogram of the second redox step is observed (Fig. 7d). This is a feature typical for the catalytic mechanisms studied under cyclo-voltammetric conditions, and it can serve as a simple criterion for recognizing the EECat mechanism, but also for distinguishing it from the EE and ECE mechanisms. Note that the voltammetric features of the first redox step at more positive potentials are not affected by the catalytic reaction (see Fig. $7 \mathrm{a}-\mathrm{d}$ ).

\section{Discussion}

In the last 15 years scores of relevant theoretical models have been presented to protein-film voltammetry, developed mainly for redox enzymes undergoing single-step electron transfer reaction under conditions of linear cyclic voltammetry [2,3,6-10]. Alongside, we started developing theoretical models for PFV under conditions of square-wave voltammetry [11-20]. In this work we present results from a theoretical study of the protein-film EE, ECE, and EECat mechanisms under conditions of cyclic staircase voltammetry. The considered redox mechanisms (EE, ECE, and EECat) are quite relevant for many flavoproteins and proteins having quinone moiety, but also for proteins containing polyvalent ions of transition metals like Mo, Mn, W, Fe or Co as redox centers. The redox chemistry of these enzymes in the biological systems usually takes place in two or more electron transfer steps [24]. Bearing in mind the complexity of the considered redox systems, it is quite useful to provide criteria for recognizing a particular redox mechanism under conditions of cyclic staircase voltammetry, but also to establish principles to distinguish it from the similar ones. When the potential difference between two electron transfer step processes is bigger than $-100 \mathrm{mV}$, then it is relatively easy to recognize and distinguish the EE from ECE and EECat, for example. To do this in a real experiment, one should only increase stepwise the concentration of the chemical agent Y or Sub (which are both redox inactive). As explained in the section of mathematical models, these variables are directly linked to the dimensionless chemical parameters $\lambda$ and $\gamma$. So, if we observe no change in the features of the recorded cyclic voltammograms by increasing the concentration of $Y$ or Sub, then the redox mechanism featuring two voltammetric processes is most probably the EE. To confirm that the studied mechanism is really EE, one should perform a scan rate analysis. If there is a link between the kinetic of the second redox process to the kinetic of the first electron transfer step, then all those facts speak about EE mechanism (Fig. 5a-d). From all three elaborated systems, the most distinguishable is, indeed, the surface EECat mechanism. If we change the concentration of the substrate Sub in the electrochemical cell, and if we observe concomitant increase of both current branches of the second redox process that get sigmoidal shapes by certain higher concentration of Sub, then those features undoubtedly belong to the EECat mechanism (see Fig. 7a-d). By the ECE mechanism, we will observe changes in both redox processes if we change the concentration of the chemical agent Y. In such a system, the first redox process will possess the features of an EC mechanism [6,14,29,30], while the second redox process at more negative potentials will have features typical for a $\mathrm{CE}$ mechanism $[6,14,15,29,30]$ (see Fig. 6a-d). Note that all mentioned analyses comprising change in the chemical agent concentration in electrochemical cell should be performed under constant scan rate.

If, however, the energy of the second electron transfer step is equal or lower than that of the first reduction process (i.e. $\Delta E^{\theta} \geq 0$ ), then a quite complicated situation exists by recognizing the nature of the studied redox mechanism. In most of the cases, this will result in existence of a single voltammetric response. The initial stage in such scenario is to identify whether the mechanism is really due to a two-step sequential electron transfer, or it is a single-step two-electron redox process. In such scenario, one can also get a help from the square-wave voltammetry. A relevant analysis that can give us hints about this is the half-peak width of the net SW 

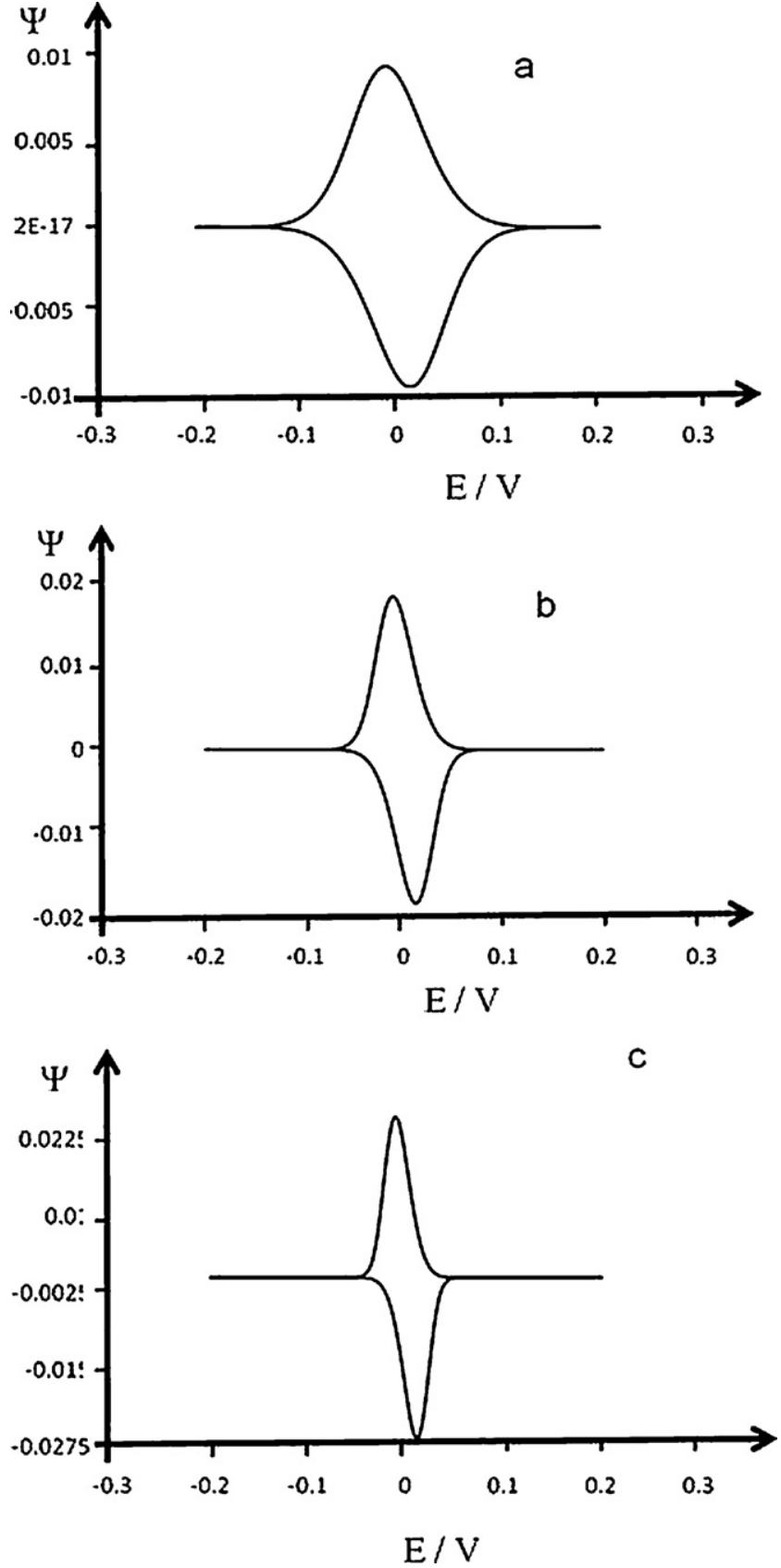

Fig. 8. Effect of the number of exchanged electrons $(n)$ to the features of simulated cyclic voltammograms in case of a simple surface redox reaction $\mathrm{A}(\mathrm{ads})+\mathrm{ne}-\leftrightarrow \mathrm{B}(\mathrm{ads})$. The number of the exchanged electrons was $n=1$ (a), 2 (b), and 3 (c). The other simulation parameters were: dimensionless kinetic parameter $K=0.1, \mathrm{~d} E=4 \mathrm{mV}, \alpha=0.5$, and $T=298 \mathrm{~K}$.

peaks [19]. The half-peak width is a parameter that is sensitive to the number of exchanged electrons also in cyclic voltammetry (see for example Fig. 8a-c), but also to the kinetics of electron transfer and $\alpha[19,29,30]$. The analysis of the half-peak width of squarewave voltammograms as a function of the frequency can provide us facts whether the studied redox process in due to a single-step or two-step sequential electron transfer [19]. Such an analysis should be performed in absence of $Y$ or Sub. By changing the concentration of Y or Sub we can get hints whether there is EE, ECE and EECat reaction going on (compare features of the voltammograms at Figs. 2-4, for example). Note that the scan rate analysis in such an experimental situation (for $\Delta E^{\sigma} \geq 0$ ) might lead us to wrong conclusions. This is because the scan rate affects both the kinetics of

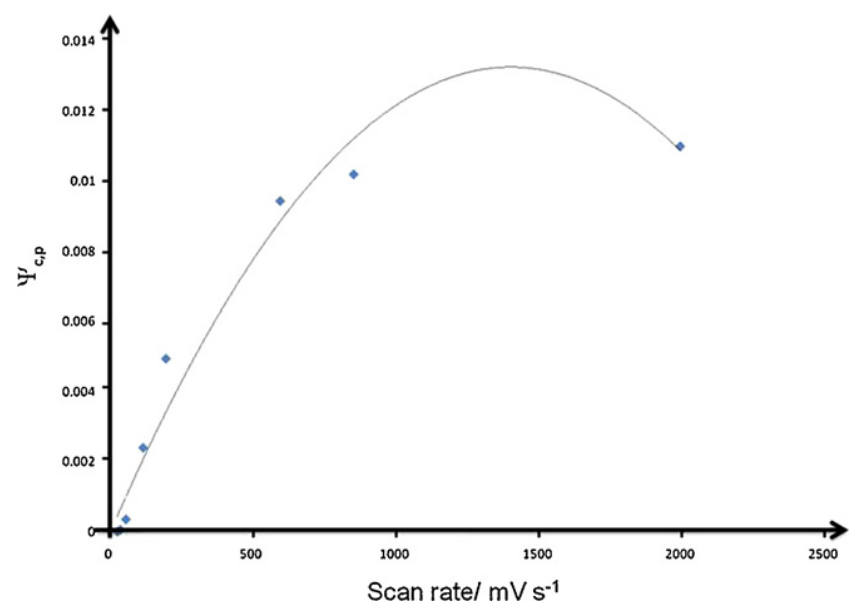

Fig. 9. Dependence of the dimensionless cathodic peak currents $\Psi_{c, p}$ on the applied scan rate obtained by simulating a simple surface redox reaction $\mathrm{A}$ (ads) + ne- $\leftrightarrow \mathrm{B}$ (ads). The number of exchanged electrons $n=1$, while the other simulation parameters were the same as those in Fig. 8.

electron transfer, but also the kinetics of the chemical reactions. The final output of the scan rate analysis in such scenario can be rather confusing.

Once we get information about the nature of the mechanism studied, the next step is to estimate the thermodynamic and kinetic parameters linked to a particular redox process. For estimating the electron transfer coefficients $\alpha$ and the standard rate constant of electron transfer of both electron transfer steps $\left(k_{\mathrm{s}, 1}\right.$ and $\left.k_{\mathrm{s}, 2}\right)$, when the two redox processes are separated for $100 \mathrm{mV}$ or more, one can use the methods proposed by Laviron [32-36] that are developed for surface redox systems in linear scan cyclic voltammetry. However, one should use the theoretical methods of Laviron with amount of caution because the cyclic staircase voltammetry is similar, but not the same with the linear cyclic voltammetry. In order proposed methods of Laviron [32-36] to be used under conditions of cyclic staircase voltammetry, one should use very small potential step (of let us say $0.5 \mathrm{mV}$ or smaller). By using bigger values for the potential step, a non-linear dependence between the peak current and the scan rate will be observed (see Fig. 9), and that is quite opposite behavior than that observed for surface redox reactions in linear cyclic voltammetry $[1,32-36]$ processes (when $\Delta E^{\Theta}<-100 \mathrm{mV}$ ) is to explore the features of so-called "quasireversible maximum". The quasireversible maximum is a typical feature of all surface redox reactions studied with pulse voltammetric techniques $[6,31]$. This is a parabolic dependence of the measured peak-currents of the voltammetric responses vs. the logarithm of the kinetic parameters related to the electron transfer steps. The maximal current is observed always by the redox reactions featuring quasireversible electron transfers (see Figs. 10a-d and 11, for example). A detailed description of the features of quasireversible maximum is provided in our previous works $[6,12,14,15,17,31]$, where we also present methods to explore this feature for estimating the kinetic of the electron transfer step. The phenomenon of "quasireversible maximum" can be used also in cyclic staircase voltammetry to estimate not only the electron transfer rate constant of the first redox step, but also that of the second redox step of all considered two-step electron mechanism (when $\Delta E^{\ominus}<$ $-100 \mathrm{mV}$ ). This can be done even in the presence of catalytic or chemical effect by ECE or EECat mechanisms. That is because the dimensionless catalytic and chemical parameters defined by EECat and ECE mechanisms do not influence the relevant features of the "quasireversibl maximum" $[6,14,17,19,31]$. It is worth to advise that no method can be applied to determine accurately the 

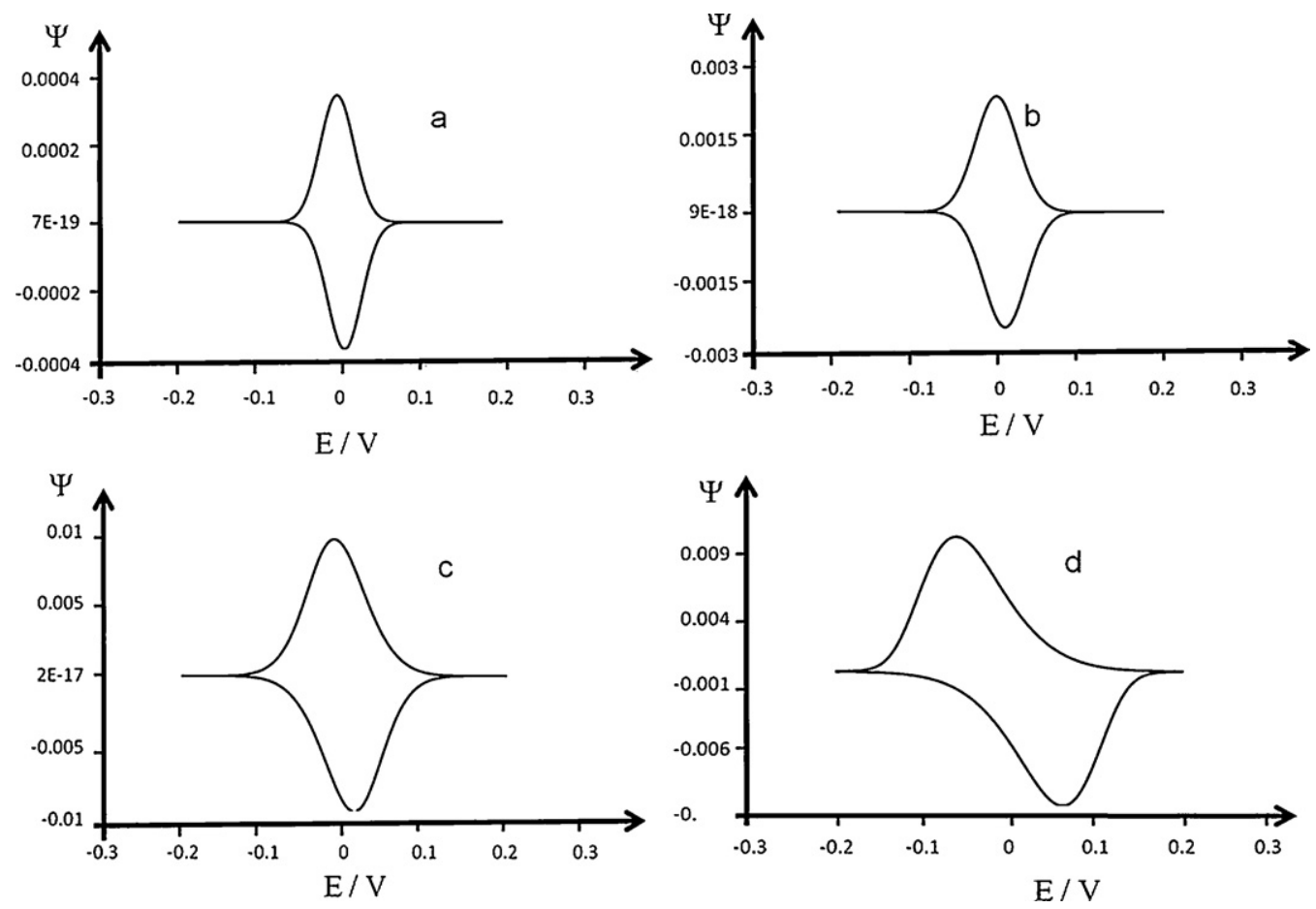

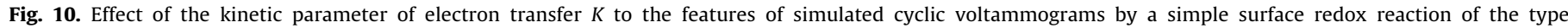

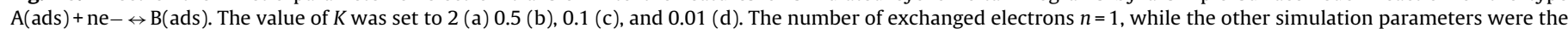
same as those in Fig. 8.

thermodynamic and kinetic parameters relevant either to the electron transfer or to the chemical steps, if both redox processes occur at the same potential and are portrayed in single cyclic voltammogram. What we will measure in such a case is a sort of mixed kinetics of electron transfer due to the inability to separate clearly both electron transfer steps. Even more complicated situation exists by the determination of the chemical kinetic parameters. This is because the high likelihood that the chemical (or catalytic) reaction by the ECE and EECat mechanism can affect not only the products of the first electron transfer steps, but also those of the second redox step. In such situation the cyclic voltammetry is not a self-sufficient method, and one should couple it with square-wave voltammetry and electrochemical impedance spectroscopy $[37,38]$ in order to get full elucidation of the relevant thermodynamic parameters of both redox steps.

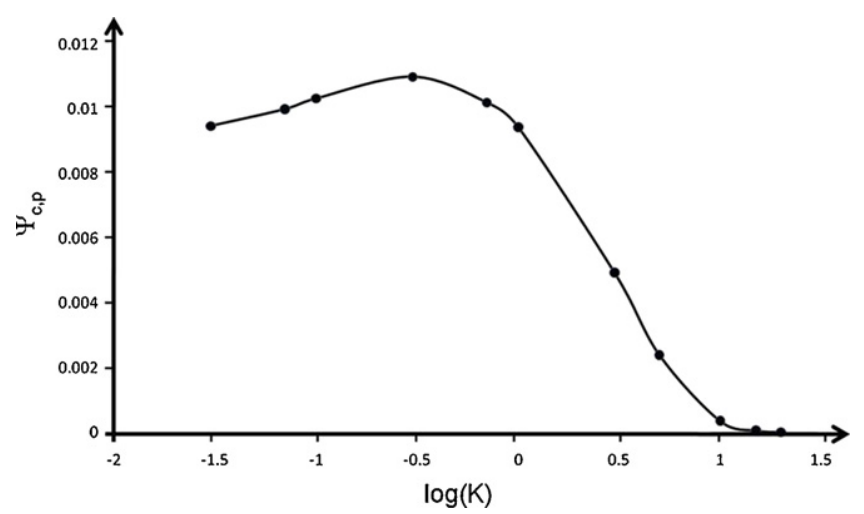

Fig. 11. Dependence of the cathodic peak currents on the logarithm of the dimensionless kinetic parameter of electron transfer $K$, simulated for $n=1$. This feature is known as a "quasireversible maximum". The other simulation parameters were the same as those in Fig. 8.

\section{Conclusions}

We present in this work the simulated results of three complex two-step redox mechanisms that are relevant to protein-film voltammetry set up. The theoretical features of the surface EE, ECE, and EECat reactions have been studied by means of cyclic staircase voltammetry. The simulated curves display the influence of the relevant physical and instrumental parameters to the features of the cyclic voltammograms. By explaining the similarities and the differences between all considered mechanisms, we give qualitative criteria to the readers for distinguishing a particular redox mechanism from the other two in case of equal and different energies of occurrence of both electron transfer steps. Moreover, we also propose simple methods for the determination of the kinetic and thermodynamic parameters relevant to the electron transfer steps and the coupled chemical reactions. The simulated mechanisms are appropriate to the redox chemistry of some enzymes containing quinone moieties or polyvalent metallic ions as redox centers.

\section{Acknowledgments}

Rubin Gulaboski thanks Alexander von Humboldt Stiftung for providing a Return postdoctoral fellowship. This work is also supported by Alexander von Humboldt Stiftung via the joint German-Macedonian project from the Research Group Linkage Programme 3.4-Fokoop-DEU/112867. The authors also acknowledge the Alexander von Humboldt Foundation for the financial support via the bilateral German-Macedonian project from the Research Group Linkage Programme 3.4-Fokoop-DEU/1128670. It is additionally acknowledged the support of DAAD foundation through multilateral project "International Masters and Postgraduate Programme in Materials Science and Catalysis" (MatCatNet) in the "Akademischer Neuaufbau Südosteuropa" programme. 


\section{References}

[1] (a) F. Marken, A. Neudeck, A.M. Bond, Cyclic voltammetry, in: F. Scholz (Ed.), Electroanalytical Methods: Guide to Experiments and Applications, SpringerVerlag, Berlin/Heidelberg, 2010;

(b) R.G. Compton, C.E. Banks, Understanding Voltammetry, World Scientific Publishing Co., Singapore, 2010.

[2] F.A. Armstrong, Voltammetry of proteins, in: A.J. Bard, M. Stratmann, G.S. Wilson (Eds.), Encyclopedia of Electrochemistry, vol. 9, Wiley VCH, Weinheim, 2002.

[3] P.N. Barlett, Bioelectrochemistry: Fundamentals, Experimental Techniques and Application, Wiley, Chichester, 2008.

[4] M.J. Eddowes, H.A.O. Hill, J. Am. Chem. Soc. 101 (1979) 4461.

[5] F.A. Armstrong, Applications of voltammetric methods for probing the chemistry of redox proteins, in: G. Lenaz, G. Milazzo (Eds.), Bioelectrochemistry: Principles and Practice, vol. 5, Birkhauser Verlag AG, Basel, 1997.

[6] R. Gulaboski, V. Mirceski, I. Bogeski, M. Hoth, J. Solid State Electrochem. (2011), doi:10.1007/s10008-011-1397-5.

[7] C. Leger, P. Bertrand, Chem. Rev. 108 (2008) 2379

[8] J. Hirst, Biochem. Biophys. Acta 1757 (2006) 225.

[9] C. Leger, S.J. Elliott, K.R. Hoke, L.J.C. Jeuke, A.K. Jones, F.A. Armstrong, Biochemistry $42(2003) 8653$.

[10] J. Hirst, F.A. Armstrong, Anal. Chem. 70 (1998) 5062.

[11] V. Mirčeski, R. Gulaboski, Electroanalysis 13 (2001) 13264

[12] V. Mirčeski, R. Gulaboski, Croat. Chem. Acta 76 (2003) 37.

[13] V. Mirčeski, R. Gulaboski, J. Solid State Electrochem. 7 (2003) 157.

[14] R. Gulaboski, J. Solid State Electrochem. 13 (2009) 1015.

[15] R. Gulaboski, V. Mirčeski, M. Lovrić, I. Bogeski, Electrochem. Commun. 7 (2005) 515.

[16] V. Mirčeski, M. Lovrić, R. Gulaboski, J. Electroanal. Chem. 515 (2001) 91.

[17] R. Gulaboski, L. Mihajlov, Biophys. Chem. 155 (2011) 1.
[18] R. Gulaboski, M. Lovrić, V. Mirčeski, I. Bogeski, M. Hoth, Biophys. Chem. 137 (2008) 49

[19] R. Gulaboski, M. Lovrić, V. Mirčeski, I. Bogeski, M. Hoth, Biophys. Chem. 138 (2008) 130.

[20] I. Bogeski, R. Gulaboski, R. Kappl, V. Mirceski, M. Stefova, J. Petreska, M. Hoth, J. Am. Chem. Soc. 133 (2011) 9293.

[21] T.J. Wydrzynski, K. Satoh, Advances in Photosynthesis and Respiration, Springer, Dordrecht, The Netherlands, 2005.

[22] J.P. McEvoy, G.W. Brudvig, Chem. Rev. 106 (2006) 4455

[23] C. Anthony, L.J. Zatman, Biochem. J. 104 (1967) 960

[24] A. Messerschmidt, R. Huber, K. Wieghardt, T. Poulos, Handbook of Metalloproteins, Wiley \& Sons, Chichester, 2001.

[25] M.L. Olmstead, R.S. Nicholson, J. Electroanal. Chem. 16 (1968) 145.

[26] R.S. Nicholson, Anal. Chem. 37 (1965) 1351.

[27] S.W. Feldberg, L. Jeftic, J. Phys. Chem. 76 (1972) 2439.

[28] A.J. Appleby, J. Electrochem. Soc. 120 (1973) 1205.

[29] M.M. Murphy, J.J. O’Dea, D. Arn, J. Osteryoung, Anal. Chem. 61 (1989) 2249.

[30] M.M. Murphy, J.J. O'Dea, D. Arn, J. Osteryoung, Anal. Chem. 62 (1990) 903.

[31] V. Mirceski, S. Komorsky-Lovric, M. Lovric, in: F. Scholz (Ed.), Square-Wave Voltammetry-Theory and Application, Springer, Berlin, 2007.

[32] E. Laviron, J. Electroanal. Chem. 105 (1979) 35.

[33] E. Laviron, E. Roullier, C. Degrand, J. Electroanal. Chem. 112 (1980) 11

[34] R. Meunier-Prest, E. Laviron, J. Electroanal. Chem. 410 (1996) 133.

[35] V. Plichon, E. Laviron, J. Electroanal. Chem. 71 (1976) 143.

[36] E. Laviron, R. Meunier-Prest, J. Electroanal. Chem. 375 (1994) 79.

[37] R. Gulaboski, C.M. Pereira, M.N.D.S. Cordeiro, I. Bogeski, E. Fereira, D. Ribeiro, M. Chirea, A.F. Silva, J. Phys. Chem. B 109 (2005) 12549.

[38] R. Gulaboski, E.S. Fereira, C.M. Pereira, M.N.D.S. Cordeiro, A. Garrau, V. Lippolis, A.F. Silva, J. Phys. Chem. C 112 (2008) 153. 